\title{
Medidas de afectos en dominios específicos*
}

Affect Measures in Specific Domains

Recibido: abril 21 de 2013 | Revisado: febrero 22 de 2014 | Aceptado: febrero 22 de 2014

\author{
Ana Paula Porto Noronha ** \\ Claudia COBÊRO *** \\ MARIANA VARANDAS DE CAMARGO \\ ROBERTA FERRAZ DE CAMPOS \\ MARIA ISABEL DE CAMPOS \\ Universidad São Francisco, Itatiba, Brasil
}

doi:10.11144/Javeriana.UPSY13-3.made

Para citar este artículo: Noronha, A. P. P., Cobêro, C., Barros, M. V. C., Campos, R. R. F., \& Campos, M. I. (2014). Medidas de afectos en dominios específicos. Universitas Psychologica, 13(3), 1005-1016. http://dx.doi.org/10.11144/Javeriana.UPSY13-3. made

* Artículo original resultado de investigación

***Correo electrónico: ana.noronha@usf.edu.br

*** Coordinadora del Curso Tecnológico en Gestión de Recursos Humanos en Facultad de Extrema.

\section{RES UMEN}

A fin de responder a la pregunta ¿Qué está bien en las personas?, la psicología positiva estudia las cualidades humanas y la promoción de su funcionamiento positivo, en particular, los afectos positivos y negativos que aluden al resultado de la intensidad y frecuencia de las emociones vivenciadas. Con el objetivo de medir los afectos en dominios específicos, a saber: yo, familia, amigos y trabajo/finanzas, el presente estudio utilizó la Escala de Afectos Zanon en una versión modificada a la que se le hizo una adaptación en su instrucción, para verificar posibles diferencias en las medidas de los afectos. Participaron de la investigación 142 personas, con edades entre 15 y 87 años, con 7.74 de nota promedio para la vida en general. Entre los principales resultados, fueron identificadas diferencias significativas entre los sexos para afectos negativos y afectos positivos y, en relación con la edad, para los afectos negativos respecto del trabajo.

Palabras clave

psicología positiva; bienestar subjetivo; evaluación psicológica; testes psicológicos

\section{A B S T R A C T}

In order to answer the question "What's right with people?" Positive Psychology aims to study the human qualities as well as promoting their positive functioning. The positive and negative affect are studied by the proposed approach and refer to the result of the intensity and frequency of experienced emotions. With the aim of measuring the affects in specific areas, namely 'I', 'Family', 'Friends' and 'Work / Finance', this study used the modified Zanon Scale Affects to detect differences in measures of affect. Participants were in the number of 142 people between 15 and 87 years old, with 7.74 average grade assigned for life in general. Among the main results, significant differences were found between genders for negative affect and positive affect, and with respect to age, there was a statistically significant negative affect toward work.

Keywords

positive psychology; subjective well-being; psychological assessment; psychological tests 


\section{Introducción}

La negligencia con que la ciencia psicológica ha tratado el estudio de los aspectos positivos y ejemplares de los seres humanos se dio, de acuerdo con Seligman (2002), en virtud del privilegio conferido a la investigación de los aspectos "anormales". A este respecto, Paludo y Koller (2007) destacan que alrededor del $70 \%$ de las personas no experimentan desórdenes mentales, al tiempo que la ausencia de enfermedad no constituye, por sí sola, felicidad o bienestar. Según Albuquerque, Martins y Neves (2008), más recientemente, la psicología ha buscado evaluar y comprender, además de los desórdenes patológicos, los aspectos positivos de la vida.

Yunes (2003) destaca que la psicología positiva puede ser definida como un movimiento para llevar a los psicólogos contemporáneos a adoptar una visión más abierta y apreciativa de los potenciales, de las motivaciones y de las capacidades humanas. De este modo, se plantean las experiencias positivas (como emociones favorables, felicidad, alegría, esperanza) y las características individuales (Marujo, Miguel Neto, Caetano \& Rivero, 2007). La pregunta central de la psicología positiva es "¿Qué está bien en las personas?”, como modo de estudiar las cualidades humanas, así como la promoción de su funcionamiento positivo. Más específicamente, la ciencia y la práctica están dirigidas a la identificación y comprensión de las cualidades y virtudes, así como a la ayuda a las personas para que tengan vidas más felices y más productivas (Snyder \& Lopez, 2009).

Entre los constructos estudiados, el Bienestar Subjetivo (BES) se ha destacado en muchas investigaciones y artículos publicados (Busseri \& Sadava, 2011). Una de las razones centrales que constituyen el BES como un concepto núcleo de la psicología positiva es el hecho de que las experiencias positivas incorporadas en un alto nivel de BES hacen la vida compensadora (Diener, Lucas \& Oishi, 2002). Para Diener, Suh, Lucas y Smith (1999), más que un constructo singular y específico, el BES representa un concepto amplio, definido como las evaluaciones cognitivas y afectivas que una persona hace sobre su propia vida, que incluyen reacciones emociona- les a eventos y juicios cognitivos de satisfacción y plenitud en diferentes dominios.

El BES, como resultado de la intensidad y frecuencia de las emociones vivenciadas por las personas, está compuesto por una dimensión afectiva (los afectos) y la satisfacción con la vida (Diener, Scollon \& Lucas, 2003; Lyubomirsky, King \& Diener, 2005). Algunos estudios proponen una estructura bidimensional para los afectos, positivos y negativos, formando dos continuos independientes de afectividad (Bradburn, 1969; Watson \& Tellegen, 1985). El orgullo, la felicidad, el entusiasmo, el encantamiento, la alegría y el afecto son las principales emociones positivas y, por otro lado, las emociones desagradables y aversivas, incluyendo tristeza, miedo, rabia, desilusión, culpa, miedo, tensión y pesimismo constituyen el afecto negativo (Diener, 1984; Novo, 2003; Watson, Clark \& Tellegen, 1988). El afecto positivo es comprendido como júbilo, altivez, alegría y éxtasis, mientras el afecto negativo puede ser representado por la vergüenza, ansiedad y preocupación, estrés, depresión y envidia, tal como lo definen Diener et al. (1999). Además, el afecto positivo caracteriza el nivel de entusiasmo de un individuo y el afecto negativo refleja una dimensión general de angustia e insatisfacción (Watson et al., 1988).

La satisfacción con la vida se subdivide en deseo de cambiar la vida, satisfacción con la vida actual, satisfacción con el pasado, satisfacción con el futuro y visión por parte de personas significativas respecto de su vida (visión de terceros). Los dominios de satisfacción incluyen la familia, la salud, el ocio, las finanzas, el self, el grupo de convivencia y el trabajo (Diener et al., 1999). De forma general, los dominios de satisfacción tienden a relacionarse con la satisfacción con la vida, sin embargo, es posible que por lo menos algunos de estos dominios representen áreas de deseo de cambio y tal vez sean fuente de baja satisfacción, mientras que otras áreas representan fuentes de plenitud y significado y sean, por tanto, fuentes de alta satisfacción (Pavot \& Diener, 2004).

Según Lyubomirsky et al. (2005), las oscilaciones de humor pueden ocurrir a intervalos de tiempo, pero la mayoría de las veces, las personas suelen notar sus niveles de afectos estables. Experimentar 
varias veces durante la vida emociones de alegría, de baja o media intensidad es más válido que experimentar pocos episodios de emociones positivas de alta intensidad; para las emociones negativas la relación es la misma (Lyubomirsky et al., 2005). En cuanto a la intensidad de los afectos, cuando los individuos experimentan altos niveles de emociones positivas, también tienden a experimentar niveles elevados de las negativas (Larsen \& Diener, 1987).

Diener y Larsen (1984) evidencian que existe una tendencia estable en la forma como el individuo percibe y vivencia los afectos. Personas con altas puntuaciones de afecto positivo experimentan episodios intensos y frecuentes de placer, considerándose alegres, entusiastas y confiados; sin embargo, estas personas también pueden sentirse tristes y afligidos en algunos momentos de su vida, no obstante, con el tiempo, hay un retorno a los niveles anteriores de afectos positivos (Diener, 1994; Watson, 2005). Por otro lado, personas con altas puntuaciones de afecto negativo experimentan repetidamente episodios intenso de desplacer, notándose tristes, desanimados y preocupados (Watson, 2005).

Las asociaciones entre BES y variables personales y contextuales fueron objeto de estudio de algunas investigaciones; por ejemplo, el ingreso no estaría relacionado con el aumento de BES, al mismo tiempo en que es previsible que los afectos positivos aumenten con la edad. Diener y BiswasDiener (2002) afirman que hay baja correlación entre indicadores económicos y BES, felicidad, satisfacción de vida y afecto positivo, de modo que el aspecto financiero por sí solo conferiría poco efecto adicional al bienestar. Además, con respecto a la edad, Lopes (2009) afirma que los afectos van evolucionando a lo largo de la vida, debido al aumento de la capacidad de regulación de las emociones con la madurez.

Las investigaciones destinadas a construir instrumentos de medida para constructos de la psicología positiva en Brasil aún son incipientes (Scorsolini-Comin \& Santos, 2010). En contexto nacional, Segabinazi et al. (2012) adaptaron una escala de afectos positivos y negativos para adolescentes. La muestra fue compuesta de 425 adolescentes que asistían a la escuela secundaria, siendo el 51\% de escuelas públicas de una ciudad del estado de Río Grande do Sul. Las edades de los participantes variaban entre 14 y 19 años $(M=16.07 ; D E=1.12)$ y $52.7 \%$ eran varones. Los autores utilizaron la Escala de Afectos Positivos y Negativos para Adolescentes, la Escala de Satisfacción de Vida Global para adolescentes y la Escala de Autoestima de Rosenberg. Sobre afecto positivo, no fue verificada interacción estadísticamente significativa entre grupo etario, sexo y escuela, grupo etario y tipo de escuela, tipo de escuela y sexo y grupo etario y sexo. Sin embargo, se verificó efecto principal de sexo $[F(1,405)=16.1 ; p$ $<0.001$ ], de modo que los varones presentaron más efectos positivos. En cuanto a afecto negativo, así como ocurrió con el positivo, hubo efecto de sexo $[F(1,408)=16.5 ; p<0.001]$, por lo que las mujeres presentaron promedios más elevados.

Alcalá, Camacho, Giner, Giner e Ibañez (2006) realizaron un estudio con el objetivo de analizar las diferentes características del afecto en función del género y de la edad. Participaron en la investigación 120 sujetos, el $57.5 \%$ mujeres, con edades comprendidas entre 18 y 50 años $(M=26.8)$. Con respecto al nivel de escolaridad, el $85.5 \%$ de los participantes informó tener estudios de nivel superior, el 11.7\% nivel secundario y el $2.5 \%$ de nivel primario. El instrumento utilizado en el estudio fue la PANAS$\mathrm{X}$, compuesto de 60 ítems con respuesta en una escala tipo Likert de cinco puntos. Todos los ítems se refieren a dos momentos: a lo largo de la vida y en las últimas semanas. Con respecto a al sexo, no se encontraron diferencias a lo largo de la vida, así como tampoco en las últimas semanas. Por otro lado, y comparando las subescalas, se encontraron escasas diferencias, presentando los varones puntuaciones más altas que las mujeres en la subescala de tristeza a lo largo de la vida y en la subescala de autoconfianza en las últimas semanas. De los 60 ítems que componen PANAS-X, a lo largo de la vida, fueron encontradas diferencias significativas entre varones y mujeres en los ítems alegre, contento y feliz, presentando las mujeres valores más elevados que los varones.

La investigación de Ferreira, Corres e Silva, Fernandes y Almeida (2008) tuvo como objetivo desa- 
rrollar una herramienta para evaluar la frecuencia de los afectos que las personas dirigen al contexto del trabajo, así como verificar su validez factorial, sus índices de precisión y su validez convergente y discriminante. La muestra estuvo compuesta por 293 empleados de organizaciones públicas y privadas, de los cuales el $40.6 \%$ eran varones y con respecto a escolaridad, el $32.8 \%$ presentaban el nivel universitario completo y el $31.4 \%$ nivel incompleto. La edad osciló entre 17 y 70 años $(M=34.89 ; \mathrm{DE}$ $=10.91)$ y el tiempo de servicio de 1 a 42 años $(M$ $=7.79 ; \mathrm{DE}=8.87)$. Los autores utilizaron tres instrumentos, a saber, Escala de Afectos en el Trabajo en el trabajo, Escala de Satisfacción en el Trabajo y Escala de Agotamiento Emocional en una sesión única de tiempo libre.

Para verificar la validez convergente y discriminante del instrumento, las puntuaciones obtenidas por cada participante en las dos dimensiones de la versión final de la Escala de Afectos en el Trabajo fueron computadas con las cinco dimensiones de las Escalas de Satisfacción con el Trabajo y de Agotamiento Emocional, indicando que todos los coeficientes fueron significativos $(p<0.01)$. Los resultados indicaron que las correlaciones fueron positivas y significativas entre los afectos positivos en el trabajo y todas las dimensiones de la Escala de Satisfacción en el Trabajo, con variaciones de $r=0.4$ a $r=-0.7$ En lo que se refiere a los afectos negativos, estos obtuvieron correlaciones negativas y significativas con todas las dimensiones de la Escala de Satisfacción en el Trabajo, variando de $r=-0.25$ a $r=-0.48$ y positivas con la Escala de Agotamiento Emocional $(r=0.69)$.

Los afectos también han sido investigados en el ambiente de trabajo con el fin de apoyar y desarrollar áreas importantes como la Gestión Estratégica. Procurando salir de las investigaciones tradicionales que se centran en las capacidades racionales de líderes y gestores, García, Sabaté y Mazagatos (2008) investigaron si las emociones de los CEO (Chief Executive Officers) en el rubro bancario tienen influencia en la forma como toman sus decisiones estratégicas. Para ello, fueron invitados a participar en la encuesta líderes de 116 empresas actuantes en el sector financiero (bancos y compañías de crédito) en España. Los investigadores recibieron 56 respuestas (el $48.3 \%$ del total) y consideraron este universo como adecuado para una encuesta de esta naturaleza. Seis de estas respuestas fueron excluidas, porque los individuos no identificaron sus instituciones o las respuestas no eran fiables. Fue utilizada la escala PANAS en su versión adaptada al idioma español. Los resultados mostraron un promedio más alto de afectos positivos $(M=42.2)$ al compararlos con el promedio de afectos negativos $(M=19.5)$, lo que era coherente con la literatura previa para otros ámbitos geográficos. Analizados en conjunto con herramientas de Gestión Estratégica, los resultados mostraron que los rasgos afectivos de los directores no están condicionados por la naturaleza jurídica y por el tamaño de las organizaciones en las que trabajan. Sin embargo, los rasgos afectivos, particularmente los afectos negativos, influyen en las decisiones estratégicas tomadas por ellos, mostrando que la manifestación de afectos negativos lleva a la selección de estrategias menos arriesgadas.

Un estudio exploratorio fue desarrollado por Noronha y Mansão (2012), con el fin de asociar intereses profesionales y afectos positivos y negativos, con una muestra de 529 estudiantes de enseñanza secundaria de tres escuelas públicas y tres privadas, ubicadas en el interior del estado de São Paulo. Del total de participantes, el 63.5\% eran estudiantes de escuelas privadas y el $35.9 \%$ del sistema público de enseñanza. La franja etaria osciló entre 14 y 17 años $(M=16 ; D E=1.48)$ y en cuanto a sexo, el $57.8 \%$ eran mujeres. Las investigadoras utilizaron el EAZ y la Escala de Orientación Profesional (EAP), para evaluar las preferencias ocupacionales. Los resultados indicaron que los participantes presentaron promedios más altos de afectos positivos, lo que indica juicio más positivo de la vida de modo general. Cabe señalar que los más altos índices de asociaciones ocurrieron entre afectos positivos con actividades burocráticas y afectos negativos con actividades relacionadas con el análisis e interpretación de datos numéricos, es decir, los afectos positivos y negativos están relacionados con intereses profesionales en diversas áreas. 
El presente estudio tuvo como objetivo investigar afectos positivos y negativos en relación con el sexo, la edad, la nota que la persona atribuyó a su vida y la asociación entre los varios dominios. Por lo tanto, las instrucciones de una escala construida y validada para Brasil fueron enriquecidas con otras informaciones, con el fin de verificar si las personas responden de manera diferente a las distintas dimensiones de su vida, como en relación con la familia, los amigos y el trabajo/finanzas. La hipótesis es que, aunque hay una tendencia estable en cómo los afectos son experimentados (Larsen \& Diener, 1984), las oscilaciones de humor pueden estar presentes (Lyubomirsky et al., 2005), siendo que para cada campo de la vida es posible asignar afectos diferentes (Diener et al., 1999).

\section{Método}

\section{Participantes}

Participaron en la encuesta 142 personas, de las cuales el $63.3 \%$ eran mujeres, con edades entre 15 y 87 años $(M=21.8 ; D E=13.8)$. En el momento de la encuesta, el 63.3\% de las personas se encontraban sin relación afectiva de pareja. Se solicitó una calificación de cero a 10 para la vida en general y las notas variaron de 2 a $10(\mathrm{M}=7.74 ; \mathrm{DE}=1.66)$.

En lo que respecta a la escolaridad, el 63.6\% de las personas correspondía a Enseñanza Secundaria Incompleta y el 89.3\% de los participantes se desempeñaban en un trabajo formal. Esto se debió a que el $71.9 \%$ de los participantes eran jóvenes de una institución de formación profesional, o sea, estaban cursando la Enseñanza Secundaria y trabajando al mismo tiempo.

\section{Instrumentos}

Se utilizó la Escala de Afectos ([EA]; Zanon \& Hutz, 2011), sin embargo, las autoras hicieron una adaptación en la instrucción de la escala para verificar las diferencias en las medidas de los afectos en diferentes dominios. A continuación, se describe la escala en su forma original y en la sección procedimientos se presentará la explicación de las modificaciones realizadas.

\section{Escala de Afectos ([EAZ]; Zanon $\mathcal{G}$ Hutz, 2011)}

La escala evalúa los sentimientos y emociones pasadas y presentes. Se compone de 20 afirmaciones, en escala del tipo Likert de cinco puntos, que van desde nada que ver contigo (1 punto) a todo que ver contigo (5 puntos). Este es un instrumento autoaplicable, en el que el respondiente solo necesita la hoja de respuesta, y se puede aplicar de forma individual o colectiva. "Me desenvuelvo con confianza en el día a día" y "Me he sentido cansado en los últimos meses" son ejemplos de ítems.

La construcción de la escala surgió de un estudio inicial en el que 853 estudiantes universitarios (57\% mujeres) con una edad promedio de 21 años $(D E=3)$, respondieron a 29 puntos, incluidos en la primera versión del instrumento, que eran sometidos a análisis de ítems, constantes en la primera versión del instrumento, que fueron sometidos a los Análisis de Componentes Principales, por rotación Oblimin. No se encontraron niveles satisfactorios de consistencias internas para el modelo de tres y cuatro factores, revelando que la mejor solución estaría compuesta de dos factores, a saber, Afectos Positivos y Afectos Negativos. La consistencia interna de la escala, evaluada mediante el alfa de Cronbach, fue de 0.83 para afecto positivo y de 0.77 para afecto negativo. Evidencias de validez convergente de la escala se verificaron a través de correlaciones del orden de 0.7 con la Positive and Negative Affect Schedule (PANAS), que evalúa afectos. Se observaron altos índices de correlación entre los afectos positivos $(r=0.73)$ y afectos negativos $(r=0.74)$, lo que indica que las subescalas de la EAZ miden el mismo constructo de las subescalas de la PANAS.

Tal como se afirmó anteriormente, se hizo una adaptación de las instrucciones de la Escala de Afectos. Así, se les pidió a los participantes que respondieran los mismos 20 ítems, con respecto a sí mismos, a la familia, a los amigos y finalmente al trabajo/finanzas. Además, se incluyó una primera sesión de identificación que contenía las iniciales del nombre, la edad, el género, la escolaridad, tipo de trabajo (formal, informal o ambos tipos de ocu- 
paciones), el estado civil y una calificación para la vida en general.

\section{Procedimiento}

El presente estudio se incluyó en un proyecto más amplio de una de las autoras. Así, después de la debida aprobación del Comité de Ética de una institución de enseñanza superior y los cuidados éticos, se inició la aplicación del instrumento.Se contactaron personas de la red de contactos de las investigadoras de una institución de formación profesional. Las aplicaciones se hicieron de forma individual o colectiva. Por último, los datos fueron pasados a una planilla del SPSS (Statistical Package for Social Sciences, versión 20), sobre la que fueron generados los resultados por medio de las estadísticas descriptivas e inferenciales.

\section{Resultados}

A fin de verificar si los diferentes dominios difieren con relación con los afectos positivos y negativos atribuidos al y', a la familia, a los amigos y al trabajo/finanzas, se procedió al test $t$ de Student, con nivel de significancia de $p=0.05$. Los resultados se presentan en la Tabla 1.

Las diferencias fueron significativas para afectos positivos para el par yo-trabajo, de modo que el promedio más alto fue en la nota atribuida al yo. En cuanto a los negativos, las significancias fueron para los pares yo en relación con los amigos y con el trabajo. En ambos casos, el promedio más alto fue atribuido al yo. Además, fueron identificadas posibles diferencias relacionadas con el sexo (Tabla 2).

$\mathrm{Al}$ analizar los resultados, se identificaron diferencias significativas para afectos negativos (yo y familia) y afectos positivos (amigos). En todas las dimensiones las mujeres presentaban los más altos promedios y el nivel de significancia fue inferior a 0.05 .

El análisis de varianza (ANOVA) fue utilizado para verificar posibles diferencias entre las edades. Considerándose la variabilidad de las edades, fue necesario separar los grupos de acuerdo con los cuartiles, de modo que resultaron tres franjas etarias. En el grupo 1 quedaron reunidos los participantes de hasta 15 años, inclusive; en el grupo 2, aquellos con edades de 16 a 21 años y en el 3, los individuos de 22 años o más. Los resultados revelaron significancia estadística apenas para los afectos negativos en relación con el trabajo $(F[142,2]=$ 11.424; $p<0.001$ ).

Por lo tanto, los grupos de edad parecen diferir cuando el contexto de los afectos negativos es el trabajo. Con el objetivo de comprender mejor dicho dato, se realizó el test de Tukey, cuyos resultados se muestran en la Tabla 3.

TABLA 1

Promedios de los afectos positivos y negativos de las dimensiones considerando los pares, valores de t y $p$

\begin{tabular}{|c|c|c|c|c|}
\hline & Promedio & $D E$ & $t$ & $p$ \\
\hline \multirow[t]{2}{*}{ AP Yo-Familia } & 40.25 & 7.339 & 1.787 & 0.076 \\
\hline & 39.33 & 7.619 & & \\
\hline \multirow[t]{2}{*}{ AP Yo-Amigos } & 40.33 & 7.327 & 1.09 & 0.278 \\
\hline & 39.71 & 7.979 & & \\
\hline \multirow[t]{2}{*}{ AP Yo-Trabajo } & 40.26 & 7.345 & 2.294 & 0.023 \\
\hline & 39.08 & 7.695 & & \\
\hline \multirow[t]{2}{*}{ AN Yo-Familia } & 28.95 & 9.543 & 1.524 & 0.13 \\
\hline & 28.06 & 9.361 & & \\
\hline \multirow[t]{2}{*}{ AN Yo-Amigos } & 28.68 & 9.339 & 6.495 & 0 \\
\hline & 23.32 & 7.677 & & \\
\hline \multirow[t]{2}{*}{ AN Yo-Trabajo } & 28.56 & 9.370 & 7.523 & 0 \\
\hline & 23.3 & 8.697 & & \\
\hline
\end{tabular}

Fuente: elaboración propia 
TABLA 2

Promedios en los diferentes contextos de la EAZ modificada considerando el sexo, valores de t y $p$

\begin{tabular}{lcccccc}
\hline & Sexo & $N$ & Prom. & DE & $p$ & $t$ \\
\hline Yo_AP & M & 47 & 39.74 & 8.339 & 0.844 & -0.197 \\
Yo_AN & $\mathrm{F}$ & 83 & 40.01 & 6.855 & & \\
Familia_AP & $\mathrm{M}$ & 49 & 25.59 & 9.251 & 0.002 & -3.185 \\
& $\mathrm{~F}$ & 83 & 30.84 & 9.094 & & \\
Familia_AN & $\mathrm{M}$ & 46 & 39.57 & 8.129 & 0.823 & 0.224 \\
& $\mathrm{~F}$ & 84 & 39.25 & 7.396 & & \\
Amigos_AP & $\mathrm{M}$ & 50 & 25.30 & 8.956 & 0.006 & -2.806 \\
& $\mathrm{~F}$ & 84 & 29.85 & 9.133 & & \\
Amigos_AN & $\mathrm{M}$ & 47 & 36.94 & 8.744 & 0.005 & -2.844 \\
& $\mathrm{~F}$ & 82 & 41.00 & 7.225 & & \\
Trabajo_AP & $\mathrm{M}$ & 49 & 23.2041 & 8.317 & 0.810 & -0.240 \\
& $\mathrm{~F}$ & 79 & 23.54 & 7.437 & & \\
Trabajo_AN & $\mathrm{M}$ & 46 & 37.44 & 8.371 & 0.089 & -1.712 \\
& $\mathrm{~F}$ & 79 & 39.85 & 7.115 & & \\
& $\mathrm{M}$ & 50 & 23.00 & 9.218 & 0.823 & -0.224 \\
\hline
\end{tabular}

AP: Afecto positivo; AF: Afecto negativo

Fuente: elaboración propia

TABLA 3

Test de Tukey para afectos negativos relacionados con el trabajo

\begin{tabular}{cccc}
\hline Grupos & $N$ & \multicolumn{2}{c}{$\alpha=0.05$} \\
\cline { 3 - 4 } & & 21.78 & \\
\hline 1 & 64 & 22.18 & \\
3 & 34 & & 29.25 \\
Sig. & 32 & 0.819 & 1 \\
\hline
\end{tabular}

Fuente: elaboración propia

Se observó la conformación de dos conjuntos. En este sentido, las personas mayores revelaron más afectos negativos que los demás participantes. Sin embargo, debe resaltarse que el promedio de edad de la muestra es de 21.8 años, por tanto, joven. Dando continuidad a la atención de los objetivos del presente estudio, se utilizó la ANOVA para verificar eventuales diferencias de promedios en relación con la escolaridad de los participantes. Estos resultados constan en la Tabla 4.

Fue identificada una diferencia significativa y otra, marginal. Más específicamente, la dimensión de afectos negativos relacionados con el trabajo se diferenció en razón del nivel de escolaridad ( $F[142$, 2] $=10.963 ; p<0.001)$ y la dimensión de afectos positivos relativos a los amigos obtuvo un coeficiente marginalmente significativo $(F[142,2]=3.033$; $p=0.52$ ). Con miras a una mejor comprensión de las diferencias identificadas, que se realizó el test de Tukey (Tabla 5).

Se observó la formación de dos conjuntos. Los participantes con nivel de escolaridad superior (completo o incompleto) se diferenciaron de aquellos con enseñanza primaria, también completa o incompleta. Se puede inferir, a partir de lo expuesto, que los participantes con niveles más altos de escolaridad presentaron más afectos negativos. La Tabla 
TABLA 4

Análisis de variancia considerando el nivel de escolaridad

\begin{tabular}{lcc}
\hline & F & p \\
\hline Yo_AP & 1.096 & 0.337 \\
Yo_AN & 1.865 & 0.159 \\
Familia_AP & 0.144 & 0.866 \\
Familia_AN & 1.718 & 0.183 \\
Amigos_AP & 3.033 & 0.052 \\
Amigos_AN & 0.591 & 0.555 \\
Trabajo_AP & 0.537 & 0.586 \\
Trabajo_AN & 10.963 & 0 \\
\hline
\end{tabular}

Fuente: elaboración propia

TABLA 5

Diferencia en la EAZ modificada para afectos negativos (trabajo) en diferentes niveles de escolaridad

\begin{tabular}{llcc}
\hline & $\mathrm{N}$ & \multicolumn{2}{c}{$\alpha=0.05$} \\
\cline { 3 - 4 } & & 1 & 2 \\
\hline Secundario & 99 & 21.78 & \\
Primario & 11 & 22.45 & 30.86 \\
Superior & 21 & & 1 \\
Sig. & & 0.962 & 1 \\
\hline
\end{tabular}

Fuente: elaboración propia

TABLA 6

Diferencia en la EAZ modificada para afectos positivos (amigos) en diferentes niveles de escolaridad

\begin{tabular}{llcc}
\hline & $N$ & \multicolumn{2}{c}{$\alpha=0.05$} \\
\cline { 3 - 4 } & 11 & 34.45 & 2 \\
\hline Primario & 96 & 39.64 & 39.64 \\
Secundario & 23 & & 41.52 \\
Superior & & 0.09 & 0.721 \\
Sig. & & & \\
\hline
\end{tabular}

Fuente: elaboración propia

6 presenta el test de Tukey para afectos positivos relacionados con los amigos.

Nuevamente, se detectó diferencia entre los niveles de escolaridad. Los participantes con nivel superior se diferenciaron de los que tenían enseñanza primaria, organizándose en conjuntos distintos. A continuación, se presentan los resultados referentes al análisis de regresión lineal (Tabla 7). Para esto, se estableció como variable dependiente cada uno de los factores evaluados por la EAZ modificada, cuando separaron en positivos y negativos. La ca- pacidad predictiva de los afectos en cada uno de sus aspectos para la nota general de la vida osciló entre el 3 y el 11\%, lo que es bajo, y el valor más bajo fue para los afectos negativos (amigos) y el más alto para afectos positivos (yo).

En cuanto a la previsión de los afectos positivos relativos al yo, el coeficiente de determinación ajustado $\left(R^{2}\right)$ fue de $0.11(F(1,131)=15.752 ; p<0.001)$. El análisis del coeficiente indica que el 11\% de la varianza fue explicada, que puede ser considerado bajo. Así, se puede inferir que otras variables influ- 
TABLA 7

Coeficientes de regresión linear para prever la nota de la vida

\begin{tabular}{lccccc}
\hline \multirow{2}{*}{\multicolumn{1}{c}{ Modelo }} & \multicolumn{2}{c}{ No estandarizados } & Coeficientes estandarizado & $t$ & $p$ \\
\cline { 2 - 6 } & $\mathrm{B}$ & Error estándar & Beta & & \\
\hline Constante (Yo-AP) & 29.055 & 2.86 & & 10.159 & 0 \\
Nota para su vida en general & 1.44 & 0.363 & 0.329 & 3.969 & 0 \\
Constante (Yo-AN) & 38.468 & 3.806 & & 10.108 & 0 \\
Nota para su vida en general & -1.246 & 0.482 & -0.22 & -2.585 & 0.011 \\
Constante (Fam-AP) & 28.346 & 3.012 & & 9.410 & 0 \\
Nota para su vida en general & 1.417 & 0.38 & 0.311 & 3.730 & 0 \\
Constante (Fam-AN) & 39.795 & 3.671 & & 10.839 & 0 \\
Nota para su vida en general & -1.521 & 0.465 & -0.272 & -3.274 & 0.001 \\
Constante (Am-AP) & 32.657 & 3.381 & & 9.66 & 0 \\
Nota para su vida en general & 0.89 & 0.424 & 0.182 & 2.097 & 0.038 \\
M & & & & & \\
Constante (Am-AN) & 29.113 & 3.281 & & 8.872 & 0 \\
Nota para su vida en general & -0.74 & 0.412 & -0.157 & -1.795 & 0.075 \\
Constante (Tra-AP) & 28.44 & 3.030 & & 9.386 & 0 \\
Nota para su vida en general & 1.386 & 0.382 & 0.308 & 3.623 & 0 \\
Constante (Tra-AN) & 33.905 & 3.612 & & 9.387 & 0 \\
Nota para su vida en general & -1.348 & 0.454 & -0.252 & -2.968 & 0.004 \\
\hline
\end{tabular}

Fuente: elaboración propia

yen en la nota que el individuo atribuye a la vida, además de los afectos positivos en relación con el yo.

El segundo y tercer valor más alto de previsión, fueron relacionados respectivamente a los afectos positivos (familia) y a los afectos positivos (trabajo), cuyos coeficientes de determinación ajustados $\left(R^{2}\right)$ fueron de $0.1(F(1,131)=13.909 ; p<0.001)$ y $0.095(F(1,125)=13.129 ; p<0.001)$. Junto a los afectos positivos relacionados con el yo, aquellos centrados en la familia y en el trabajo parecen ser los más fuertes, aunque bajos, para explicar la nota que el individuo atribuye a la vida.

\section{Discusión}

La problemática del presente estudio fue construida con base en las afirmaciones de Diener y Larsen (1984), Lyubomirsky et al. (2005) y Diener et al. (1999), en el sentido de que oscilaciones de humor pueden estar presentes y que a cada campo de la vida es posible atribuir afectos distintos. En particular, los últimos autores defienden que, en relación con la satisfacción con la vida, familia, salud, ocio, finanzas, self, como ejemplos, son campos con características específicas, por lo que es posible que algunos sean fuentes de menos satisfacción que otros.

En cuanto a las diferencias de medias, los resultados revelaron significancia en tres de los seis pares, siendo que el promedio para el yo fue el más alto, reafirmando la concepción de los autores de que dominios específicos pueden despertar sensaciones de placer o desplacer, de felicidad o descontento. Las dimensiones trabajo y amigos fueron las que revelaron las diferencias significativas; para familia no fue observada, tanto en relación con los afectos positivos, como negativos. Replicando los hallazgos de Garcia Sabaté y Mazagatos (2008), los afectos positivos fueron superiores a los negativos.

Con el fin de entender mejor los resultados, se investigaron además posibles diferencias relacionadas con el sexo. A este respecto, las mujeres presentaron promedios más altos para yo, familia, cuando se investigaron los afectos negativos, y amigos, en el caso de los positivos. Segabinazi et al. (2012), al estudiar jóvenes con promedio de edad de 16 años aproximadamente, identificaron que los chicos 
presentaron más afectos positivos y Alcalá et al. (2006), en la encuesta con adultos, encontraron que los hombres obtuvieron puntuaciones más altas en la subescala Tristeza a lo largo de la vida y Aautoconfianza. Por lo tanto, se puede afirmar que los resultados del presente estudio son más semejantes a los de Segabinazi et al. (2012).

Las diferencias entre las edades también fueron objeto de investigación. Para el dominio de trabajo hubo diferencia significativa, y los mayores presentaron más afectos negativos que los demás. En cuanto a este hecho, los afectos positivos no se comportaron de forma diferente en función de la edad, lo que no corrobora los hallazgos de Lopes (2009). El autor afirmó que los afectos positivos aumentaban a lo largo de la vida, debido a la capacidad de regulación de las emociones con la madurez. Sin embargo, hay que señalar que los participantes del presente estudio, en general, eran jóvenes, lo que sugiere que los datos sean analizados con cautela.

La escolaridad fue otra variable estudiada. Los afectos negativos en relación con el trabajo y los positivos referentes a los amigos se diferenciaron, de modo que personas con más escolaridad superior (nivel superior) presentaron más afectos negativos y positivos en relación con los dominios citados (trabajo y amigos, respectivamente). Estos hallazgos deben ser aclararse mejor. En cuanto al dato, Diener y Biswas-Diener (2002) defienden la baja asociación entre indicadores económicos y felicidad, satisfacción de vida y afecto positivo.

El análisis de regresión fue utilizado para evaluar el poder predictivo de los afectos positivos y negativos en las varias dimensiones propuestas por el instrumento modificado en relación con la nota atribuida a la vida. De modo general, el poder predictivo parece ser bastante bajo. Los afectos positivos relación con el yo, aquellos centrados en la familia y en el trabajo parecen ser los más fuertes, para explicar la nota que el individuo atribuye a la vida. A continuación, se presentan las consideraciones finales. En alguna medida, lo encontrado era esperado, ya que BES está compuesto de afectos y satisfacción con la vida y, aunque la nota atribuida pudiera revelar satisfacción con la vida, deben considerarse otras dimensiones como familia, salud, ocio, finanzas, self, grupo de convivencia y trabajo (Diener et al., 1999; Diener et al., 2003; Lyubomirsky et al., 2005).

En síntesis, se pretende responder a la cuestión problematizada en el presente artículo. Para Pavot y Diener (2004), los dominios de afectos se diferencian, puesto que algunos pueden generar más satisfacción que otros. Los afectos relativos al yo se muestran como los más fuertes en los varios análisis realizados, lo cual es consistente con las aseveraciones de Lyubomirsky et al. (2005). Estos autores afirman que es más probable que el orgullo, la felicidad, el entusiasmo, el encantamiento, la alegría y el afecto estén frecuentemente más presentes que las emociones desagradables y aversivas, incluyendo la tristeza y la rabia (Diener, 1984; Novo, 2003; Watson, Clark \& Tellegen, 1988).

Como limitación del presente estudio, se debe considerar que la muestra investigada se conformó por conveniencia, sin los cuidados con la estratificación y análisis de otras variables. Además, el instrumento utilizado para la recogida de datos fue adaptado. Se requieren, por lo tanto, verificaciones de validez para el nuevo formato.

\section{Referencias}

Albuquerque, F. J. B., Martins, C. R., \& Neves, M. T. S. (2008). Bem-estar subjetivo emocional e coping em adultos de baixa renda de ambientes urbano e rural. Estudos em Psicologia, 25(4), 509-516.

Alcalá, V., Camacho, M., Giner, D., Giner, J., \& Ibañez, E. (2006). Afectos y género. Psicothema, 18(1), 143-148.

Bradburn, N. M. (1969). The structure of psychological well-being. Chicago: Aldine.

Busseri, M. A., \& Sadava, S. W. (2011). A review of the tripartite structure of subjective well-being: Implications for conceptualization, operationalization, and synthesis. Personality and Social Psychology Review, 15(3), 290-314.

Diener, E. (1984). Subjective well-being. Psychological Bulletin, 95(3), 542-575. 
Diener, E. (1994). Assessing subjective well-being: Progress and opportunities. Social Indicators Research, 31(2), 103-157.

Diener, E., \& Biswas-Diener, R. (2002). Will money increase subjective well-being? A literature review and guide to needed research. Social Indicators Research, 57(2), 119-169.

Diener, E., \& Larsen, R. J. (1984). Temporal stability and cross-situational consistency of affective, behavioral, and cognitive responses. Journal of Personality and Social Psychology, 47(4), 871-883.

Diener, E., Lucas, R. E., \& Oishi S. (2002). Subjective well-being: The science of happiness and life satisfaction. En C. R. Snyder \& S. J. Lopez (Eds.), Handbook of positive psychology (pp. 63-73). NewYork: Oxford University Press.

Diener, E., Scollon C. N., \& Lucas, R. E. (2003). The envolving concept of subjective well-being: The multifaceted nature of happiness. Advances in Cell Aging and Gerontology, 15, 187-219.

Diener, E., Suh, E. M., Lucas, R. E., \& Smith, H. L. (1999). Subjective well-being: Three decades of progress. Psychological Bulletin, 125(2), 276-302.

Ferreira, M. C., Correa e Silva, A. P., Fernandes, H. A. \& Almeida, S. P. (2008). Desenvolvimento de validação de uma escala de afetos no trabalho (ESAFE). Avaliação Psicológica, 7(2), 143-150.

García, J. B. D., Sabaté, J. M. F., \& Mazagatos, V. B. (2008). Las emociones y la dirección: icondicionan los afectos de los directivos sus decisiones estratégicas? Universia Business Review, 17, 26-39.

Larsen, R. J., \& Diener, E. (1987). Affect intensity as an individual difference characteristic: A review. Journal of Research in Personality, 21(1), 1-39.

Lopes, S. A. S. (2009). Envelhecimento, bem-estar subjectivo e prática desportiva: um estudo realizado em adultos praticantes de natação e hidroginástica. Dissertação de Mestrado, Programa de Pós Graduação em Ciência do Desporto, Área de especialização em Actividade Física e Saúde, Faculdade de Desporto da Universidade do Porto, Brasil.

Lyubomirsky, S., King, L., \& Diener, E. (2005). The benefits of frequent positive affect: Does happiness lead to success? Psychological Bulletin, 131(6), 803-855.
Marujo, H. A., Miguel Neto, L., Caetano, A., \& Rivero, C. (2007). Revolução positiva: psicologia positiva e práticas apreciativas em contextos organizacionais. Comportamento Organizacional e Gestão, 13(1), 115-136.

Noronha, A. P. P., \& Mansão, C. S. M. (2012). Interesses profissionais e afetos positivos e negativos: estudo exploratório com estudantes de ensino médio. Psico-USF, 17(2), 323-331.

Novo, R. F. (2003). Para além da Eudaimonia. O bem-estar psicológico em mulheres na idade adulta avançada [Textos Universitários de Ciências Sociais e Humanas]. Lisboa: Fundação Calouste Gulbenkian.

Paludo, S. S., \& Koller, H. (2007). Psicologia positiva: uma nova abordagem para antigas questões. Paidéia, 17(36), 9-20.

Pavot, W., \& Diener, E. (2004). Findings on subjective well-being: Applications to public policy, clinical interventions, and education. En A. P. Linely \& S. Joseph (Eds.), Positive psychology in practice (679692). Hoboken, NJ: Wiley.

Scorsolini-Comina, F., \& Santos, M. A. (2010). Psicologia positiva e os instrumentos de avaliação no contexto brasileiro. Psicologia: Reflexão e Crítica, 23(3), 440-448.

Segabinazi, J. D., Zortea, M., Zanon, C., Bandeira, D. R., Giacomoni, C. H., \& Hutz, C. S. (2012). Escala de Afetos Positivos e Negativos para Adolescentes: adaptação, normatização e evidências de validade. Avaliação Psicológica, 11(1), 1-12.

Seligman, M. (2002). Positive psychology, positive prevention, and positive therapy. En C. R. Snyder \& S. J. Lopez (Eds.), Handbook of positive psychology (pp. 3-9). New York: Oxford University Press.

Snyder, C. R., \& Lopez, S. J. (2009). Psicologia positiva: uma abordagem científica e prática das qualidades humanas. Porto Alegre: Artmed.

Watson, D. (2005). Rethinking the mood and anxiety disorders: A quantitative hierarchical model for DSM-V. Journal of Abnormal Psychology, 114(4), 522-536.

Watson, D., \& Tellegen, A. (1985). Toward a consensual structure of mood. Psychological Bulletin, 98(2), 219-235.

Watson, D., Clark, L. A., \& Tellegen, A. (1988). Development and validation of brief measures of positive 
Ana Paula Porto Noronha Fagundes, Claudia Cobêro, Mariana Varandas de

Camargo Barros, Roberta Ramazotti Ferraz de Campos, Maria Isabel de Campos

and negative affect: The PANAS scales. Journal of Personality and Social Psychology, 54(6), 1063-1070.

Yunes, M. A. M. (2003). Psicologia positiva e resiliência: o foco no indivíduo e na família [Eejemplar especial]. Psicologia em Estudo, 8, 75-84.
Zanon, C., \& Hutz, C. S. (2011). Construção de uma Escala de Afetos e suas Relações com os Cindo Grande Fatores da Personalidade (Big Five). Tese de Doutorado, Programa de Pós-Graduação em Psicologia do Desenvolvimento, Universidade Federal do Rio Grande do Sul, Rio Grande do Sul, Brasil. 